\title{
Development and evaluation of BGO fiber scintillator
}

\section{Takayuki Yanagida, Mariya Zhuravleva, and Akira Yoshikawa}

IMRAM, Tohoku University

2-1-1, Katahira, Aoba-ku, Sendai, Japan

E-mail: t_yanagi@tagen.tohoku.ac.jp

\section{Kei Kamada}

Material reserch laboratoly, Furukawa, Co. Ltd.,

1-25-13, Kannondai, Tukuba, Ibaragi, 305-0856, Japan

\section{Valery Chani}

Department of Materials Science \& Engineering, McMaster University

1280 Main Street West, Hamilton, Ontario, L8S 4L7, Canada

In high energy physics, an imaging detector which consists of multi anode PMT (MAPMT) and pixel scintillators having a size of several mm square is used. In this kind of detector, a spatial resolution is limited by a size of scintillator pixel, because scintillator arrays which have size of sub-mm square are difficult to be made. Today, our group successfully developed single crystal growth technology of BGO fiber with a $100 \mu \mathrm{m}$ and $100 \mathrm{~mm}$ length by using the micro-pullingdown method. When coupled with APD and irradiated by gamma-ray radio isotopes of ${ }^{22} \mathrm{Na}$ and ${ }^{137} \mathrm{Cs}, 511 \mathrm{keV}$ and $662 \mathrm{keV}$ photo-absorption peaks are clearly obtained, respectively.

International workshop on new photon-detectors PD07

Kobe University, Kobe, Japan

27-29 June, 2007 


\section{Introduction}

Scintillators have played a major role as a gamma-ray detector for the last century. In this century, gamma-ray detectors need a high spatial resolution, because most of applications are for radiation imaging. For example in PET (Positron Emission Tomography), $\sim 5 \mathrm{~mm}$ spatial resolution is achieved by arrayed pixel scintillators coupled with MAPMT (Multi Anode PMT). In such applications, now, a limit of the spatial resolution is a size of scintillator pixels, because recent light detectors have a sub-mm intrinsic spatial resolution (e.g., [1]).

In order to couple these sub-mm light detectors, we developed a fiber BGO crystal scintillator, and evaluate its gamma-ray responses, coupled with Si avalanche photo-diode (hereafter APD), because most of sub-mm detectors are Si semiconducters (PSAPD, SSD, DSSD).

\section{Experiment}

We developed single crystal growth technology of BGO fiber with a $100 \mu \mathrm{m}$ and $100 \mathrm{~mm}$ length by using the micro-pullingdown method, as shown in figure 1 . At this time, a cross section of the fiber is a circle, and the shape is changeable by using other crucibles. After developing the fiber, we mounted it on the PMT (H 6521) with Silicone grease (OKEN, 6262A). The measurement temperature is controlled at $-20 \pm 1$ degrees Celsius by a heat bath, to decrease leak current of APD. In order to collect scintillation photons, we covered them with ESR film (3M). Then, we irradiated ${ }^{22} \mathrm{Na}$ and ${ }^{137} \mathrm{Cs}$.

\section{Results and Conclusion}



Figure 1. Thin fiber BGO scintillator sample. The outer case is a normal $\mathrm{CD} / \mathrm{DVD}$ case.

Figure 2 shows a gamma-ray spectrum of BGO fiber, irradiated by ${ }^{22} \mathrm{Na}$ and ${ }^{137} \mathrm{Cs}$. As represented in this figure, $511 \mathrm{keV}$ and $662 \mathrm{keV}$ photo-absorption peaks are clearly detected. Also, $32.2 \mathrm{keV}$ photo peak from ${ }^{137} \mathrm{Cs}$ was directly detected by APD. Generally, the peak created by Si direct detection shows a good energy resolution, however, the peak from a scintillator becomes wide because of a smaller signal to noise ratio. The reason why $1.275 \mathrm{MeV}$ peak from ${ }^{22} \mathrm{Na}$ is not seen is attributed to a very small dimension of the present BGO fiber sample. Generally, as exemplified in some works (e.g., [2]), to obtain a clear photo peak from a particular shaped scintillator is difficult.. From these results, the conclusion is that we can develop inorganic fiber crystal scintillators, and they actually works as a scintillator. 




Figure 2. Gamma-ray spectra of ${ }^{22} \mathrm{Na}$ and ${ }^{137} \mathrm{Cs}$, where photo peaks are represented by arrows. The horizontal axis shows MCA channel, and the vertical count rate.

\section{References}

[1] Kanai., S.,, Shar., et al., IEEE Trans. Nucl. Sci., 49 (2002) 1687

[2] T. Itoh, and T. Yanagida, et al., NIM-A, 579 (2007), 239 